\title{
Universalism Entails Extensionalism
}

\author{
Achille C. Varzi \\ Department of Philosophy, Columbia University, New York
}

[Final version published in Analysis, 69 (2009), 599-604]

1. Universalism (also known as Conjunctivism, or Collectivism) is the thesis that mereological composition is unrestricted. More precisely:

(U) Any non-empty collection of things has a fusion, i.e., something that has all those things as parts and has no part that is disjoint from each of them. ${ }^{1}$

Extensionalism is the thesis that sameness of composition is sufficient for identity. More precisely:

(E) No two things have exactly the same proper parts (unless they are atomic, i.e., have no proper parts at all).

Clearly these two theses are not equivalent. They are, however, more closely related than one might think. For while (E) does not entail (U), the converse entailment holds - or so I will argue. More precisely, the entailment holds as long as it is agreed that the following postulates are constitutive of the meaning of 'part':

(1) Transitivity: Any part of any part of a thing is itself part of that thing.

(2) Supplementation: Whenever a thing has a proper part, it has at least another part that is disjoint from the first.

2. One way to establish the entailment can be extracted from two results of Simons (1987: 29ff), which concern a set of postulates logically equivalent to (1) and (2). The first is that such postulates license the derivation of (E) from the following strengthening of (2):

(3) Strong Supplementation: Whenever a thing is not part of another, the first has at least a part that is disjoint from the the second.

${ }^{1}$ I write 'is disjoint from' as shorthand for 'has no parts in common with'. I will also write 'overlaps' for 'has parts in common with' and 'is a proper part of' for 'is part of, but not identical to'. 
The second is that they license the derivation of (3) itself from the following thesis:

(4) Product: Any pair of overlapping things has a product, i.e., something whose parts are exactly the parts those things have in common.

Now, obviously (U) entails (4), for a product of any two overlapping things is just a fusion of their common parts: it has each of those parts as parts and it has no further part, hence no part that is disjoint from each of them. Thus, given (1) and (2), it follows that (U) entails (3) (by Simons's second result), hence that it entails (E) (by the first).

There is, however, a more direct argument for the same conclusion, and it serves well to spell it out to see that the detour via (3) and (4) is unnecessary. Indeed, this second argument does not even rely on the full strength of (U); all that is needed, to get $(\mathrm{E})$, is finitary Universalism, i.e., the assumption that any pair of objects has a fusion. For suppose that $x$ and $y$ are distinct non-atomic objects. There are two possibilities:

(a) One object, say $x$, is part of the other, $y$. Then obviously $x$ and $y$ don't have the same proper parts. For $x$ must be a proper part of $y$, though it cannot be a proper part of itself (on pain of being non-self-identical).

(b) Neither $x$ nor $y$ is part of the other. Then let $z$ be a fusion of $x$ and $y$, whose existence follows from (U). By definition of 'fusion', $x$ must be part of $z$, in fact a proper part. (If $x$ were identical to $z$, then $y$, which must also be part of $z$, would be part of $x$, contrary to what we are assuming.) Thus, by (2), $z$ has a part, $z_{1}$, that is disjoint from $x$. By definition of 'fusion', again, $z_{1}$ cannot be also disjoint from $y$, which is to say that it must have a part, $z_{2}$, in common with $y$. Now, either $z_{2}$ is a proper part of $y$, or $z_{2}$ is $y$. In the first case, it is clear that $z_{2}$ cannot be part of $x$, let alone a proper part, since $x$ is disjoint from $z_{1}$. In the second case, let $z_{3}$ be a proper part of $y$ (which is not atomic). By (1), $z_{3}$ must be part of $z_{1}$, hence again it cannot be part of $x$, let alone a proper part. It follows that in both cases $x$ does not have the same proper parts as $y$.

Since (a)-(b) are the only possibilities, this shows that the non-identity of $x$ and $y$ is reflected in their different mereological composition. (E) now follows by generalization.

3. Several philosophers, including myself, think that both Universalism and Extensionalism are necessarily true, so this result will leave them unmoved. ${ }^{2}$ However, just as there are philosophers who accept Extensionalism while rejecting Univer-

\footnotetext{
${ }^{2}$ For the record, I defend Universalism in (2006a) and Extensionalism in (2008).
} 
salism (e.g., Chisholm 1976), others may be inclined to accept Universalism while rejecting Extensionalism. That is, they may hold that any old collection of things has a fusion (for instance, because they agree with Lewis 1986 that restricted composition entails ontic indeterminacy, which they reject) while maintaining that distinct objects may have the same proper parts (for instance, because they agree with Wiggins 1980 that the existence and persistence conditions of an object depend on the organization of its parts and not just on the parts themselves: a cat may outlast a "mere" fusion of its body cells; or because they agree with Sanford 2003 that the existence and persistence conditions of a "mere" fusion are themselves dependent on the things it fuses: a fusion of all cat-molecules may exist when there is no fusion of all cats, since the molecules may outlast the cats). To such philosophers, the entailment from (U) to (E) must come as bad news.

There are, of course, two ways of resisting the entailment on their behalf. One would simply consist in denying (1) or (2), on which the argument depends. The other would consist in denying that $(\mathrm{U})$ provides an adequate formulation of Universalism. I don't have much to say about the first option. Personally, I would dismiss it altogether if 'part' is to signify what it is meant to signify. Even Simons (1987), by himself a non-universalist non-extensionalist, does not hesitate to regard (1) and (2) as constitutive of the meaning of the word-axioms that capture what there essentially is to any relation if it is to count as parthood at all (in contrast to (U) and (E), which would reflect substantive ontological theses). But this is not the place to embark in a defense of this view. ${ }^{3}$ If one's notion of parthood is so different from the standard notion as to violate either (1) or (2), then the meaning of (U) and (E) would change accordingly and the relationship between such theses might well be up for grabs. The second option, however, is worth examining.

To say that Universalism is not properly captured by (U) is to say that it rests on the wrong notion of fusion. And surely enough there are some alternatives. For example, Lewis (1991: 2) says that the fusion of all cats understood as in (U), i.e.,

(f) something that has all cats as parts and has no part that is disjoint from every cat could equally be defined as

$\left(f^{\prime}\right)$ something that overlaps all and only those things that overlap some cat.

Lewis is speaking of the fusion of all cats, so he is already assuming Extensionalism. And he is right: in the presence of (E), (f) and $\left(f^{\prime}\right)$ are equivalent. Yet the

${ }^{3}$ For the record, I defend (1) in (2006b). As for (2), I simply go along with Simons, although I appreciate that more might be needed. (See e.g. Smith 2009.) 
anti-extensionalist is free to consider $\left(\mathrm{f}^{\prime}\right)$ as a genuine alternative, and there is no a priori reason to think that it should yield an equivalent formulation of Universalism. In fact, it is the notion of fusion exemplified by $\left(\mathrm{f}^{\prime}\right)$ that appears in many classical treatments of mereology, whether extensional (as in Leonard and Goodman's original Calculus of Individuals, 1940) or not (as in Rescher 1955). It is also customary to construe a fusion as a minimal upper bound relative to parthood, so that a fusion of all cats would be

$\left(f^{\prime \prime}\right)$ something that is part of all and only those things that have all cats as parts.

This is, for instance, the notion of fusion used in Bostock (1979) and typically featured in the linguistic literature on the semantics of plurals and mass nouns (see Landman 1991). Lewis himself sometimes speaks as though $\left(f^{\prime \prime}\right)$ would be just as fine as (f), but that's because he is assuming (U). As it turns out, if a collection of things has an (f)-type fusion, then this is ipso facto an ( $\left.\mathrm{f}^{\prime \prime}\right)$-type fusion of the same things. Yet the converse need not hold, and one is certainly free to consider the latter notion as a genuine alternative in the characterization of Universalism.

Now, it is good news for the non-extensionalist that these notions are indeed not equivalent even if (1)-(2) are granted. And it may be good news that both alternatives to (f) yield variants of Universalism that are in fact compatible with the rejection of Extensionalism. However, the good news ends here. For closer inspection reveals that in each case the outcome has intolerable consequences.

Regarding $\left(\mathrm{f}^{\prime}\right)$, consider the model diagrammed below, where proper parthood relations are represented by lines going upwards:

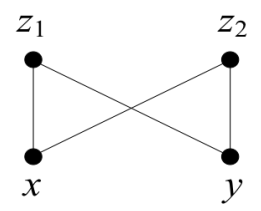

This model satisfies (1) and (2) but not (E), since $z_{1}$ and $z_{2}$ are distinct in spite of having the same proper parts, $x$ and $y$. Indeed, both $z_{1}$ and $z_{2}$ count as fusions of $x$ and $y$ in the original sense exemplified by (f) as well as in the alternative sense exemplified by $\left(\mathrm{f}^{\prime}\right)$. However, there is nothing here that has both $z_{1}$ and $z_{2}$ as parts, so those two elements do not in turn have an (f)-type fusion, which is to say that (U) fails along with (E) (as expected). Do $z_{1}$ and $z_{2}$ have an ( $\left.\mathrm{f}^{\prime}\right)$-type fusion? One should expect the answer to be in the negative, too. Yet, as noted in Hovda (2009) (and it is somewhat surprising that this had never been noticed before), the answer is in the affirmative. There are, in fact, two ( $\left.\mathrm{f}^{\prime}\right)$-type fusions of $z_{1}$ and $z_{2}$. One is $z_{1}$ itself, since $z_{1}$ overlaps every element in the model and, a fortiori, every element 
overlaps either $z_{1}$ or $z_{2}$. The other is $z_{2}$, for the same reason. This is hard to swallow. For no matter how exactly one defines the word, one should always expect a fusion to include, among its parts, all the things it fuses. ${ }^{4}$ And it gets worse. For, on closer look, $z_{1}$ also counts as an ( $\left.\mathrm{f}^{\prime}\right)$-type fusion of $z_{2}$, and $z_{2}$ of $z_{1}$. This is simply intolerable. No matter how exactly one defines the word, surely a fusion is supposed to include, among its parts, at least some of the things it fuses. So, yes, we have a model in which Extensionalism fails even though every non-empty collection of things has a "fusion" of some sort, but the price we are asked to pay is to change the meaning of the word beyond any reasonable limit.

What about $\left(\mathrm{f}^{\prime \prime}\right)$ ? In this case, the corresponding notion of fusion does ensure - by definition - that a fusion always include the things it fuses among its parts, as with (f). This means that the above remarks do not apply, since the four-element model is not closed under this sort of fusion: $z_{1}$ and $z_{2}$ have no upper bound. However, suppose we add the missing elements, as in the following diagram:

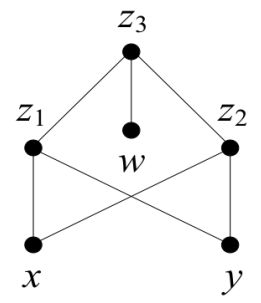

Note that the middle, disjoint element $w$ must be added along with $z_{3}$ in order to satisfy (2), Supplementation. Now $z_{3}$ counts as an ( $\left.\mathrm{f}^{\prime \prime}\right)$-type fusion of $z_{1}$ and $z_{2}$, though not as an (f)-type or an $\left(\mathrm{f}^{\prime}\right)$-type fusion (precisely because of the additional element $w$ ), and inspection shows that every non-empty collection of elements has a fusion in this sense- - minimal upper bound. That's the good news for the universalist non-extensionalist, since the model is still in violation of (E). Yet, again, something is clearly amiss here. For $z_{3}$ is not just composed of $z_{1}$ and $z_{2}$; it is also composed of $w$, which is disjoint from both. In fact, $z_{3}$ also counts as an ( $\left.\mathrm{f}^{\prime \prime}\right)$-type fusion of the two atoms $x$ and $w$ (leaving out $y$ ) and of the two atoms $y$ and $w$

${ }^{4}$ Pontow (2004) notes that the model in question is also a counterexample to the "standard" characterization of classical extensional mereology given by Simons (1987: 37) and inherited by much literature that followed, according to which the theory is axiomatized by adding the ( $\left.\mathrm{f}^{\prime}\right)$-variant of $(\mathrm{U})$ to a "basis" consisting of Transitivity and Supplementation plus Reflexivity (everything is part of itself) and Antisymmetry (no two things are part of each other). I made the same mistake elsewhere, so I take this opportunity to rectify: only the (f)-based version of (U) does the job (and Antisymmetry turns out to be redundant). 
(leaving out $x$ ). All this is hard to swallow. No matter how exactly one defines the word, surely a fusion is supposed to be entirely composed of the things it fuses. Of course, $z_{3}$ is also an $\left(\mathrm{f}^{\prime \prime}\right)$-type fusion of all of $z_{1}, z_{2}$ and $w$, or of all three atoms $x, y$, and $w$, but that is little consolation. And it gets worse. For we could expand the model by splitting $w$ into two further atoms, $w_{1}$ and $w_{2}$. Then $z_{3}$ would count as an $\left(\mathrm{f}^{\prime \prime}\right)$-type fusion of, say, the two atoms $x$ and $w_{1}$ and also of the two atoms $y$ and $w_{2}$. That is simply intolerable. No matter how exactly one defines the word, surely one should not get the same fusion from two wholly disjoint classes of atoms. In short, once again we have a non-extensional model in which every non-empty collection of things has a "fusion" of some sort, but the price we are asked to pay is to change the meaning of the word beyond acceptability.

I conclude that replacing the (f)-type notion of fusion in (U) with either of its $\left(f^{\prime}\right)$-type or $\left(f^{\prime \prime}\right)$-type variants cannot, in the present context, be claimed to result in a reasonable formulation of Universalism, let alone a better formulation. This sort of way out is simply a no-go. On pain of foregoing Transitivity (1), or Supplementation (2), the only reasonable formulation of Universalism will carry in its wake a commitment to Extensionalism.

\section{REFERENCES}

Bostock D., 1979, Logic and Arithmetic, Volume 2, Oxford: Clarendon.

Chisholm R. M. 1976, Person and Object. A Metaphysical Study, La Salle: Open Court.

Hovda P. 2009, 'What Is Classical Mereology?', Journal of Philosophical Logic 38: 55-82.

Landman F. 1991, Structures for Semantics, Dordrecht: Kluwer.

Leonard H. S. and Goodman N. 1940, 'The Calculus of Individuals and Its Uses', Journal of Symbolic Logic 5: 45-55.

Lewis D. K. 1986, On the Plurality of Worlds, Oxford: Blackwell.

Lewis D. K. 1991, Parts of Classes, Oxford: Blackwell.

Pontow C. 2004, 'A Note on the Axiomatics of Theories in Parthood', Data \& Knowledge Engineering 50: 195-213.

Rescher N. 1955, 'Axioms for the Part Relation', Philosophical Studies 6: 8-11.

Sanford D. 2003, 'Fusion Confusion', Analysis 63: 1-4.

Simons P. M. 1987, Parts. A Study in Ontology, Oxford: Clarendon.

Smith D. 2009, 'Mereology without Weak Supplementation', Australasian Journal of Philosophy, in press.

Varzi A. C. 2006a, 'The Universe Among Other Things', Ratio 19: 107-120.

Varzi A. C. 2006b, 'A Note on the Transitivity of Parthood', Applied Ontology 1: 141-146.

Varzi A. C. 2008, 'The Extensionality of Parthood and Composition', Philosophical Quarterly 58: 108-133.

Wiggins D. 1980, Sameness and Substance, Oxford: Blackwell. 